Check for updates

Cite this: RSC Adv., 2019, 9, 29765

Received 17th July 2019

DOI: 10.1039/c9ra05517g

rsc.li/rsc-advances
Accepted 26th August 2019

\section{Studies on PBFMO-b-PNMMO alternative block thermoplastic elastomers as potential binders for solid propellants $\dagger$}

\author{
Minghui Xu, (DD *ab Xianming Lu, ${ }^{\mathrm{b}}$ Hongchang Mo, ${ }^{\mathrm{b}}$ Ning Liu, (D) ${ }^{\mathrm{b}}$ Qian Zhang ${ }^{\mathrm{b}}$ \\ and Zhongxue $\mathrm{Ge}^{\mathrm{b}}$
}

An energetic polymer, poly[3,3-bis(2,2,2-trifluoro-ethoxymethyl)oxetane]glycol-block-poly(3nitratomethyl-3-methyloxetane) (PBFMO-b-PNMMO), was synthesized using poly[3,3-bis(2,2,2-trifluoroethoxymethyl)oxetane]glycol (PBFMO) and poly(3-nitratomethyl-3-methyloxetane) (PNMMO) as the raw materials and toluene diisocyanate as the coupling agent via a prepolymer process. The structures of the energetic polymers were characterized by Fourier transform infrared spectroscopy (FT-IR), ${ }^{1} \mathrm{H}$ nuclear magnetic resonance spectrometry $\left({ }^{1} \mathrm{H}-\mathrm{NMR}\right)$, and ${ }^{13} \mathrm{C}$ nuclear magnetic resonance spectrometry $\left({ }^{13} \mathrm{C}\right.$ NMR). A universal testing machine, scanning electron microscopy (SEM), differential scanning calorimetry (DSC) and thermogravimetric analysis (TGA) were used to investigate the mechanical properties and thermal behavior of PBFMO- $b$-PNMMO. The PBFMO- $b$-PNMMO-based elastomers exhibited superior mechanical properties (10.54 MPa, 723\%) in comparison with PNMMO (6.18 $\mathrm{MPa}, 635 \%)$ and good compatibility with $\mathrm{HMX}$ and Al. The glass transition temperature $\left(T_{\mathrm{g}}\right)$ and decomposition temperature $\left(T_{\mathrm{d}}\right)$ were found to be -20.4 and $220{ }^{\circ} \mathrm{C}$, respectively. The cook-off results indicated that the PBFMO-bPNMMO/Al compositions could release significantly more heat than the PNMMO/Al compositions. Therefore, PBFMO- $b$-PNMMO may serve as a promising energetic binder for future solid propellant formulations.

\section{Introduction}

The development of high-performance composite propellants is a key area of research within current solid-state rocket propulsion. In order to attain a satisfactory performance, the replacement of the traditional inert hydroxylterminated polybutadiene (НTPB) binder with energetic binders has attracted an increasing interest. ${ }^{1-3}$ Compared with inert binders, energetic binders contain energetic groups such as the azide group $\left(-\mathrm{N}_{3}\right)$, nitrate esters $(-\mathrm{O}-$ $\left.\mathrm{NO}_{2}\right)$, nitramines $\left(-\mathrm{N}-\mathrm{NO}_{2}\right)$, and $\mathrm{C}-$ nitro groups $\left(-\mathrm{C}-\mathrm{NO}_{2}\right)$, which can not only impart additional energy to the formulations, but also improve the overall oxygen balance of the propellant. ${ }^{4,5}$ Among the energetic binders, azide functional polymers (viz., glycidyl azide polymer (GAP), poly(3,3-bis(3azido methyl)oxetane) (PBAMO), poly((3-azido methyl)-3methyl oxetane) (PAMMO)) and nitrato polyethers (viz., poly((3-nitrato methyl)-3-methyl oxetane) (PNMMO), and

\footnotetext{
${ }^{a}$ State Key Laboratory of Fluorine \& Nitrogen Chemicals, Xi'an 710065, China ${ }^{b} X i{ }^{\prime} a n$ Modern Chemistry Research Institute, Xi'an 710065, China. E-mail: mhuixu@ 163.com; Tel: +862988291306

$\dagger$ Electronic supplementary information (ESI) available. See DOI: $10.1039 / \mathrm{c} 9 \mathrm{ra05517g}$
}

poly(glycidyl nitrate) PGN) have received widespread atten-

In the past three decades, fluoropolymers have been extensively studied in propellant or plastic-bonded explosive (PBX) formulations..$^{9-11}$ This is due to their high density, long-term chemical stability, low coefficients of friction, and compatibility with energetic materials, especially strong oxidative ability with metals. ${ }^{12-14}$ For example, the magnesium, Teflon, and Viton (MTV) system, which was wellknown in decoys and flares, has high specific infrared radiant intensity and especially large specific reaction energy of $9.4 \mathrm{~kJ} \mathrm{~g}^{-1}$ in comparison to TNT and RDX with the yields of just $3.72 \mathrm{~kJ} \mathrm{~g}^{-1}$ and $6.569 \mathrm{~kJ} \mathrm{~g}^{-1}$, respectively. ${ }^{15}$ Therefore, fluoropolymers may serve as promising polymeric binders in metal-rich solid propellant formulations.

In this study, an energetic thermoplastic elastomer, poly [3,3-bis(2,2,2-trifluoro-ethoxymethyl)oxetane]glycol-blockpoly(3-nitratomethyl-3-methyloxetane) (PBFMO- $b$-PNMMO), was prepared via a prepolymer process for metal fuel-rich solid propellant formulations. PBFMO- $b$-PNMMO consisted of poly(3-nitratomethyl-3-methyloxetane) as the soft segment and TDI-extended PBFMO as the hard segment. The molecular structure of PBFMO- $b$-PNMMO was characterized by FT-IR and NMR. The mechanical properties and thermal behavior of the elastomer were investigated by tion in recent decades. ${ }^{6-8}$ 
tensile tests, scanning electron microscopy (SEM), differential scanning calorimetry (DSC) and thermogravimetric analysis (TGA). Moreover, the cook-off test was used to evaluate the thermal performance between PBFMO- $b$ PNMMO and Al.

\section{Experimental}

\subsection{Materials}

Butane diol (BDO), dichloromethane (DCM), ethanol and 1,2dichloroethane were purchased from Sinopharm Chemical Reagent Co. Ltd (Xi'an, China). $\mathrm{BF}_{3}$-dimethyl ether $\left(\mathrm{BF}_{3} \mathrm{OEt}_{2}\right)$, dibutyltin dilaurate (DBTDL), and toluene diisocyanate (TDI) were supplied by Chengdu Kelong Chemical Reagents Company. $\mathrm{BDO}$ and $\mathrm{BF}_{3} \mathrm{OEt}_{2}$ were distilled under reduced pressure prior to use. All solvents for the reactions were of analytical grade and were dried before use. [3,3-Bis(2,2,2trifluoroethoxymethyl)oxetane]glycol (BFMO) was synthesized following the published procedure. ${ }^{\mathbf{1 6}}$ (3-Nitrato methyl)-3methyl oxetane (NMMO) was synthesized as we described previously. ${ }^{17,18}$

\subsection{Synthesis of PNMMO by cationic ring-opening polymerization}

PNMMO was synthesized via the cationic ring-opening polymerization of NMMO using BDO as the initiator. Briefly, BDO $(0.54 \mathrm{~g}, 6 \mathrm{mmol})$ and $\mathrm{BF}_{3} \mathrm{OEt}_{2}(0.52 \mathrm{~g}, 3.6 \mathrm{mmol})$ were dissolved in DCM $(20 \mathrm{~mL})$ in a flame-dried $250 \mathrm{~mL}$ three necked flask with a mechanical stirrer, thermometer and condenser under argon atmosphere and stirred for $0.5 \mathrm{~h}$. A solution of NMMO (29 g, $0.18 \mathrm{~mol})$ in DCM $(10 \mathrm{~mL})$ was then added dropwise into the reaction solution within a period of $6 \mathrm{~h}$, and the reaction was allowed to proceed for $24 \mathrm{~h}$ at $35{ }^{\circ} \mathrm{C}$. Subsequently, the reaction was stopped by the addition of $2 \%$ sodium bicarbonate solution. The organic phase was washed by distilled water, dried over sodium sulphate, and filtered, and the solvent was evaporated off in vacuum at $40^{\circ} \mathrm{C}$ to recover $28.2 \mathrm{~g}$ of PNMMO $(95.5 \%$, yield). GPC analysis: $M_{\mathrm{n}}=4650 \mathrm{~g} \mathrm{~mol}^{-1}$, polydispersity index $(\mathrm{PDI})=1.18$ (against polystyrene standards).

\subsection{Polymerization of PBFMO}

PBFMO was also synthesized through cationic ring-opening polymerization. The typical reaction procedure was as follows: BDO (0.54 g, $6 \mathrm{mmol}), \mathrm{BF}_{3} \mathrm{OEt}_{2}(0.52 \mathrm{~g}, 3.6 \mathrm{mmol})$ and $26 \mathrm{~mL}$ DCM were charged into a three necked flask fitted with a mechanical stirrer and condenser under argon atmosphere and stirred for $0.5 \mathrm{~h}$. BFMO solution (42.3 g BFMO in $10 \mathrm{~mL}$ DCM) was then added dropwise to the reaction flask and the polymerization was done at room temperature $\left(30^{\circ} \mathrm{C}\right)$ for $24 \mathrm{~h}$. After polymerization, the reaction was ended by the addition of $2 \%$ sodium bicarbonate solution, followed by washing with distilled water. After evaporation of the solvent and drying under vacuum, the product was received as a white wax polymer (yield: $97.6 \%$ ). GPC analysis: $M_{\mathrm{n}}=6840 \mathrm{~g} \mathrm{~mol}^{-1}$, PDI $=1.22$ (polystyrene standards).

\subsection{Synthesis of PBFMO- $b$-PNMMO-based thermoplastic elastomers}

PBFMO- $b$-PNMMO-based thermoplastic elastomers were synthesized using PNMMO and PBFMO as raw materials and TDI as a coupling agent via a prepolymer process. The typical reaction procedure was as follows: exactly $8 \mathrm{~g}$ PBFMO was dissolved in $15 \mathrm{~mL}$ freshly distilled 1,2-dichloroethane under high pure argon (99.99\%) at $60{ }^{\circ} \mathrm{C}$ for $0.5 \mathrm{~h}$. DBTDL $(20 \mu \mathrm{L})$ and TDI $(0.85 \mathrm{~g})$ dissolved in $10 \mathrm{~mL}$ 1,2-dichloroethane was added to this mixture and stirred at $80{ }^{\circ} \mathrm{C}$ for $2 \mathrm{~h}$ until the hydroxyl group could not be detected. Then, PNMMO (16 g) dissolved in $15 \mathrm{~mL}$ 1,2-dichloroethane was added and polymerization was allowed to proceed for another $2 \mathrm{~h}$ at $80{ }^{\circ} \mathrm{C}$. Thereafter, the reaction mixture was cooled to room temperature and slowly added to ethanol under stirring. The crude product precipitated out was redissolved in DCM and purified by precipitation in $n$-pentane. The recovered polymer was finally dried under vacuum at $40{ }^{\circ} \mathrm{C}$ to give a yellowish, elastomeric compound. GPC analysis: $M_{\mathrm{n}}=$ $53135 \mathrm{~g} \mathrm{~mol}^{-1}$, PDI $=1.82$ (polystyrene standards).

\subsection{Measurements}

FTIR spectra were recorded in a Bruker Tensor 27 instrument in the range of 4000-650 $\mathrm{cm}^{-1}$. Nuclear magnetic resonance (NMR) spectra were measured on a Bruker $500 \mathrm{MHz}$ instrument. Gel permeation chromatography (GPC) was conducted on Water GPC using tetrahydrofuran as the mobile phase and polystyrene standards for calibration. Mechanical properties including tensile strength and elongation at break of elastomer films were measured on an AG-X universal testing machine with a tensile rate of $50 \mathrm{~mm} \mathrm{~min}^{-1}$, and a mean value of five replicates from each film was taken. SEM was carried out on a VEGA 3 LMU scanning electron microscope (TESCAN, Czech Republic). All the elastomer films were frozen in liquid nitrogen and then snapped immediately. The fracture surfaces of the fractured films were sputtered with gold and then photographed. Differential scanning calorimetry (DSC) was carried out in a DSC Q1000 equipment at a heating rate of $10^{\circ} \mathrm{C} \mathrm{min}{ }^{-1}$ under argon atmosphere. Thermogravimetric analysis (TGA) was performed with an SDT Q600 TGA instrument between 25 and $500{ }^{\circ} \mathrm{C}$ at a heating rate of $10{ }^{\circ} \mathrm{C} \mathrm{min}^{-1}$. A cook-off test was carried out on a special equipment designed by the Institute of Chemical Material, as described in Fig. 1. Samples were sealed

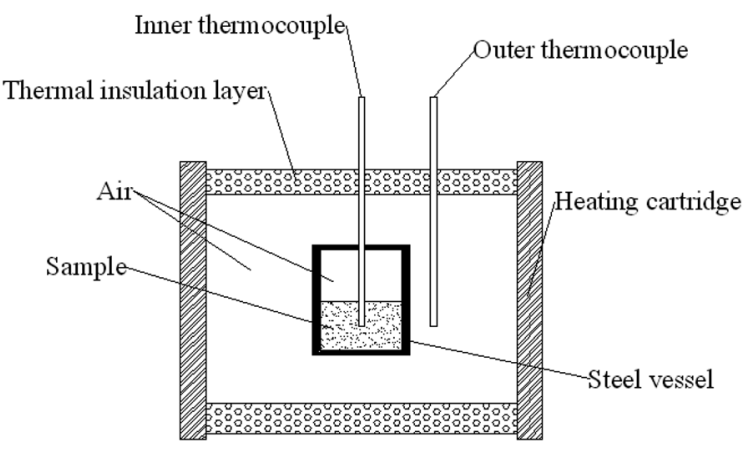

Fig. 1 Schematic geometry of cook-off test. 
with a heating rate of $1{ }^{\circ} \mathrm{C} \mathrm{min}^{-1}$ from room temperature to $220^{\circ} \mathrm{C}$, and the changes in temperatures from inner and outer thermocouples with time were recorded.

\section{Results and discussion}

\subsection{Synthesis of PBFMO-b-PNMMO-based thermoplastic elastomers}

PBFMO- $b$-PNMMO-based thermoplastic elastomers were synthesized using PBFMO and PNMMO as the raw materials and TDI as the coupling agent via a prepolymer process; the synthetic route is described in Fig. 2. The structure of the assynthesized PBFMO- $b$-PNMMO was confirmed by FTIR and NMR. Fig. 3 shows the FTIR spectra of PNMMO and PBFMO- $b$ PNMMO, whereas Fig. 4(A and B) depicts the ${ }^{1} \mathrm{H}$ NMR and ${ }^{13} \mathrm{C}$ NMR spectra of PBFMO- $b$-PNMMO. As shown in Fig. 3 , the characteristic absorption peaks at 1632, 1278 and $870 \mathrm{~cm}^{-1}$ correspond to $-\mathrm{O}-\mathrm{NO}_{2}$ from $\mathrm{PNMMO}{ }^{19}$ The appearance of strong peaks at 1225 and $1161 \mathrm{~cm}^{-1}$ was attributed to $-\mathrm{CF}_{3},{ }^{20-22}$ the appearance of peaks at $1412-1534 \mathrm{~cm}^{-1}$ was attributed to the characteristic absorption of TDI, and the characteristic absorption peaks at 3311,1710 and $1533 \mathrm{~cm}^{-1}$ certified the formation of urethane linkage. ${ }^{23,24}$ Therefore, these results gave strong evidence that the prepolymer process indeed occurred by the formation of PBFMO- $b$-PNMMO.

NMR analysis was used to determine the real composition of the as-synthesized PBFMO- $b$-PNMMO, which is shown in Fig. 4. The signals at 3.75 and $4.4 \mathrm{ppm}$ were attributed to PNMMO, ${ }^{19}$ and the signal at $3.26 \mathrm{ppm}$ was attributed to PBFMO. The corresponding carbon signals of the methylene carbons from PBFMO and PNMMO appeared at 73.6 and 75 ppm. The signal at $40.4 \mathrm{ppm}$ was attributed to the quaternary carbon atom of $\mathrm{C}$ $\mathrm{O}-\mathrm{NO}_{2}$. The signal at $2.18 \mathrm{ppm}$ belonged to the methylene protons of BDO, which is the initiator of PNMMO and PBFMO; the corresponding carbon signal appeared at $26.4 \mathrm{ppm}$. The signals at $1.57 \mathrm{ppm}$ and 6.3-8 ppm were attributed to the methyl protons and methine protons on the benzene rings of TDI, respectively, and the corresponding carbon signals appeared at

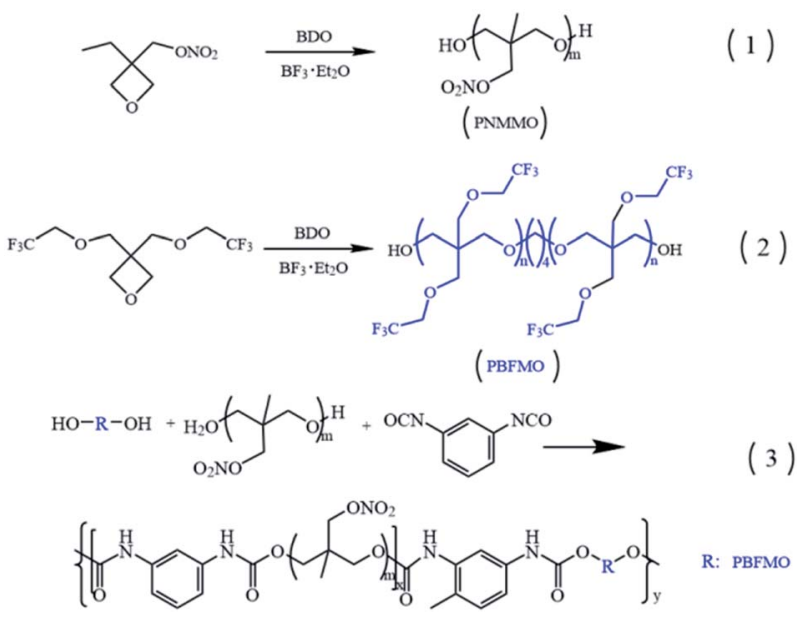

Fig. 2 The synthesis route of PBFMO-PNMMO-ETPE.

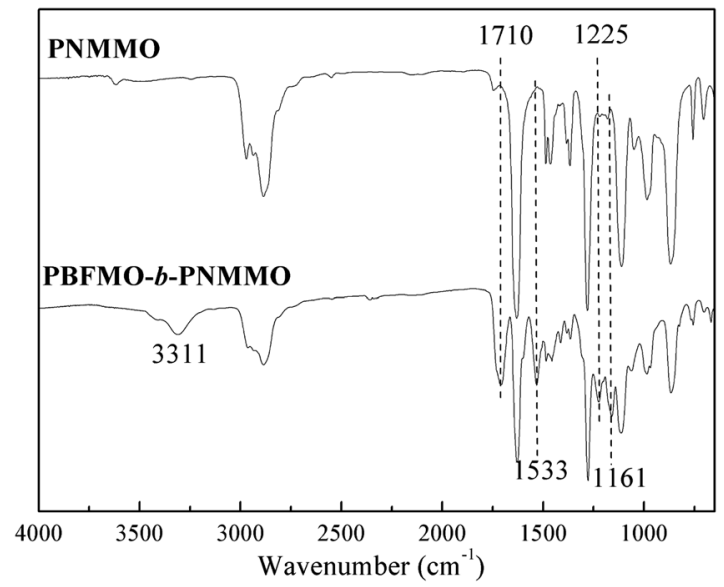

Fig. 3 FTIR spectra of PNMMO and PBFMO- $b-\mathrm{PNMMO}$.

17.1 ppm and 120-135 ppm. By comparing the GPC traces of PBFMO, PNMMO and PBFMO- $b$-PNMMO (Fig. S1 $\dagger$ ), a significant increase in the molecular weight was observed, which provided an experimental proof of the successful formation of PBFMO- $b$-PNMMO.

\subsection{Mechanical properties of PBFMO-b-PNMMO-based thermoplastic elastomers}

The mechanical properties of the PBFMO- $b$-PNMMO- and PNMMO-based thermoplastic elastomers were evaluated via a universal testing machine by measuring the tensile
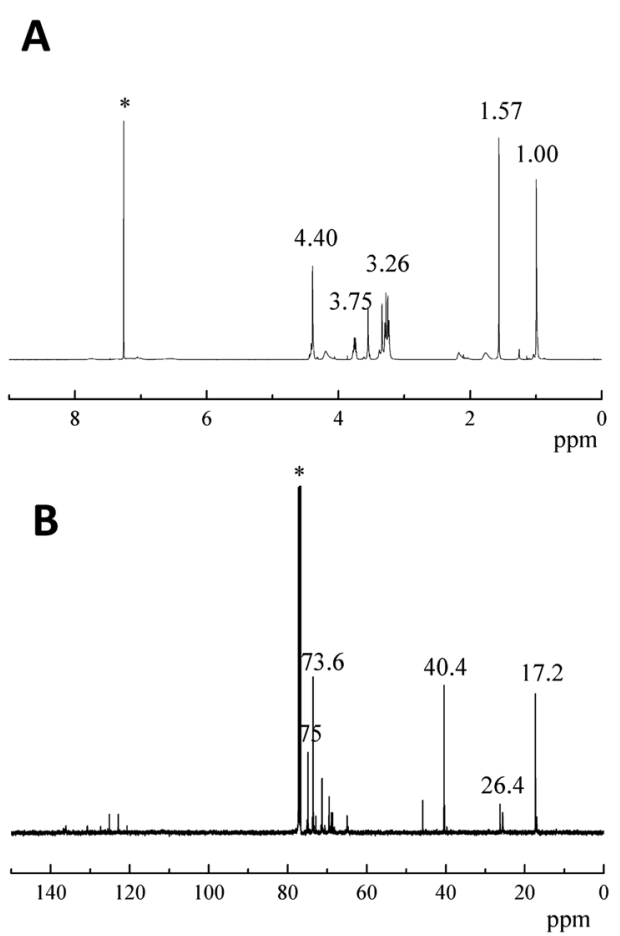

Fig. $4{ }^{1} \mathrm{H}-\mathrm{NMR}$ spectrum $(\mathrm{A})$ and ${ }^{13} \mathrm{C}-\mathrm{NMR}$ spectrum (B) of PBFMO-bPNMMO in $\mathrm{CDCl}_{3}$. 
strength and breaking elongation..$^{25,26}$ An overlay of the stress-strain curves of PBFMO- $b$-PNMMO- and PNMMObased thermoplastic elastomers is shown in Fig. 5. In contrast with the tensile strength of the PNMMO-based thermoplastic elastomers, the tensile strength of PBFMO$b$-PNMMO significantly increased from $6.18 \mathrm{MPa}$ to $10.54 \mathrm{MPa}$, while the breaking elongation increased from $635 \%$ to $723 \%$. In theory, the change in tensile strength is usually opposite to that of breaking elongation. However, it was worth noting that the maximum value of the tensile strength for PBFMO- $b$-PNMMO was obviously higher than that for the control group, while the breaking elongation was still maintained. It is well-known that thermoplastic elastomers consist of hard segments, which act as fillers and physical crosslinks, and soft segments, which are in a rubbery state that leads to flexibility. The introduction of PBFMO having superior crystallinity provided strong crosslinking points, which inhibited the plastic deformation of the PNMMO-based soft segments and ensured an increase in the tensile strength of PBFMO- $b$-PNMMO. Meanwhile, the amounts of crosslinking points remained constant, resulting in similar values of elongation at break. Therefore, PBFMO- $b$-PNMMO exhibited satisfactory mechanical properties.

\subsection{Fracture morphologies of PBFMO- $b$-PNMMO-based thermoplastic elastomers}

To investigate the difference in the mechanical properties of the films prepared from PBFMO- $b$-PNMMO and PNMMO-based ETPE, the fracture morphologies of the films were studied by SEM. Fig. 6 shows the SEM images of the films prepared from PBFMO- $b$-PNMMO (A) and PNMMO-based ETPE (B). As shown in Fig. 6, in comparison with the observation for PNMMO-based ETPE, the fractured stripes of the gel prepared from PBFMO- $b$ PNMMO are few and scattered and the fractured surface gradually becomes smooth. Furthermore, micrographs also revealed no obvious two-phase structures in the PBFMO- $b$-PNMMO films. This indicates that PNMMO and PBFMO have good miscibility and compatibility. ${ }^{27}$

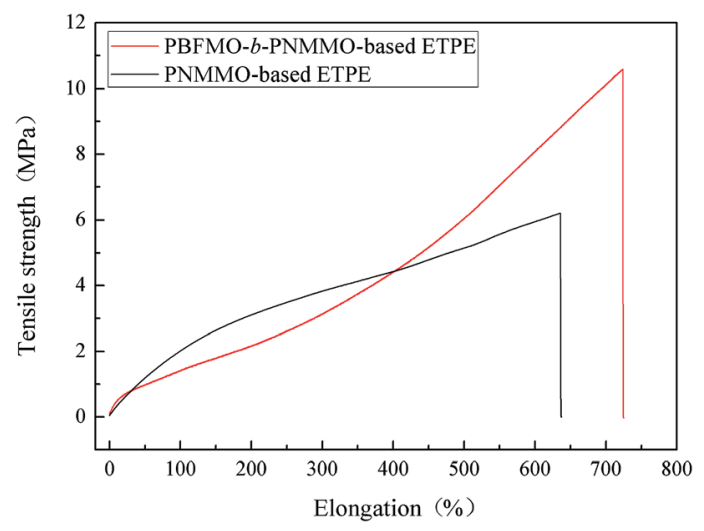

Fig. 5 Tensile testing of films prepared from PBFMO- $b-P N M M O$ and PNMMO-based ETPE.
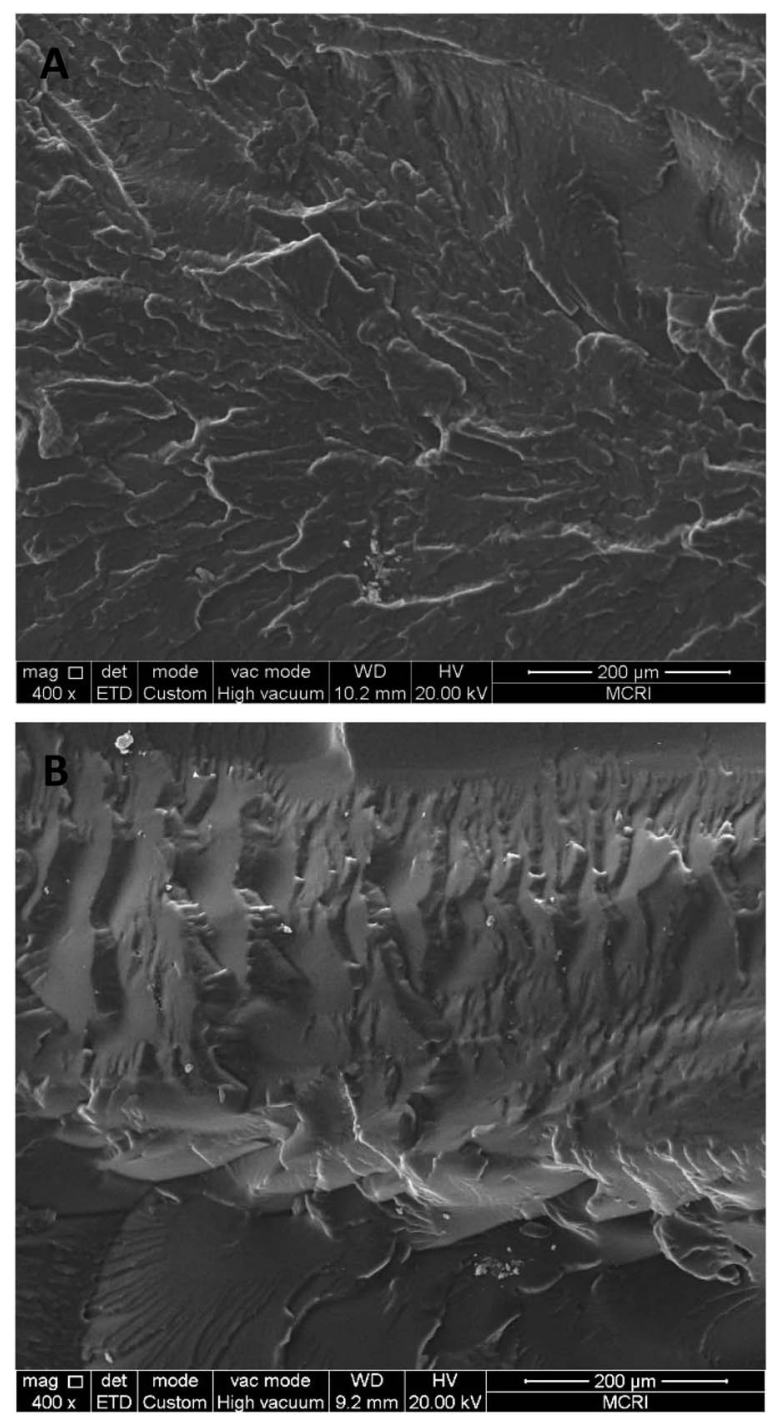

Fig. 6 SEM images for the fracture surface of the films prepared from PBFMO-b-PNMMO (A) and PNMMO-based ETPE (B).

\subsection{Glass transition temperature}

Glass transition temperature $\left(T_{\mathrm{g}}\right)$ is one of the most important properties of polymeric binders because it determines the processing and application temperature range. ${ }^{28,29}$ Differential scanning calorimetry (DSC) was used to measure the glass transition temperature of PBFMO- $b$-PNMMO, as shown in Fig. 7. As segmented block polyurethane, PBFMO- $b$-PNMMO is expected to possess two different $T_{\mathrm{g}}$ values. However, the curve shown in Fig. 7 exhibits only one $T_{\mathrm{g}}$ of $-20.4{ }^{\circ} \mathrm{C}$. This may be due to the fact that the PNMMO and PBFMO segments are welldispersed and blended within the films, which are also shown in Fig. 6; thus, polyurethane consists of one phase. The same phenomenon was also observed in other literatures.,.$^{\mathbf{3 , 6 , 3 0}}$

\subsection{Thermal decomposition}

It is well-known that thermal stability is a very important and crucial property for the application of energetic binders. ${ }^{31,32}$ 


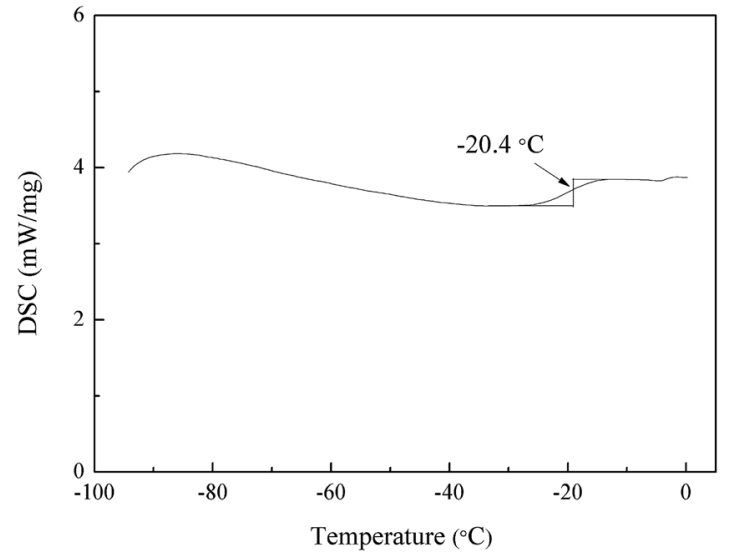

Fig. 7 Low temperature DSC curve of PBFMO- $b$-PNMMO under nitrogen.

Thus, both DSC and TGA were applied to study the thermal stability of PBFMO- $b$-PNMMO. The DSC curve (Fig. 8) shows exothermic decomposition maximum at $220^{\circ} \mathrm{C}$, which is due to the exothermic scission of nitrate esters. As shown in Fig. 9, the TGA and derivative thermogravimetry (DTG) curves of PBFMO$b$-PNMMO also reflect excellent resistance to thermal decomposition up to $200{ }^{\circ} \mathrm{C}$. The first weight loss temperature at $209{ }^{\circ} \mathrm{C}$ corresponded to the exothermic decomposition peak in the DSC curve. The second and third main weight losses at $304{ }^{\circ} \mathrm{C}$ and $456{ }^{\circ} \mathrm{C}$ were due to the main-chain thermal decomposition of PBFMO- $b$-PNMMO. In any case, both the DSC and TGA results confirm that PBFMO- $b$-PNMMO starts to degrade at high temperatures, thus showing satisfactory thermal stability.

\subsection{Cook-off test}

A slow cook-off test was performed to study the thermal performance of PBFMO- $b$-PNMMO and Al powders. ${ }^{33-35}$ The cook-off curves of thermoplastic elastomers/Al compositions (Fig. 10) show an initial linear curves, subsequent impetuous curves and ultimately linear curves again. Generally, the integral of the outer and inner temperature curves can evaluate the

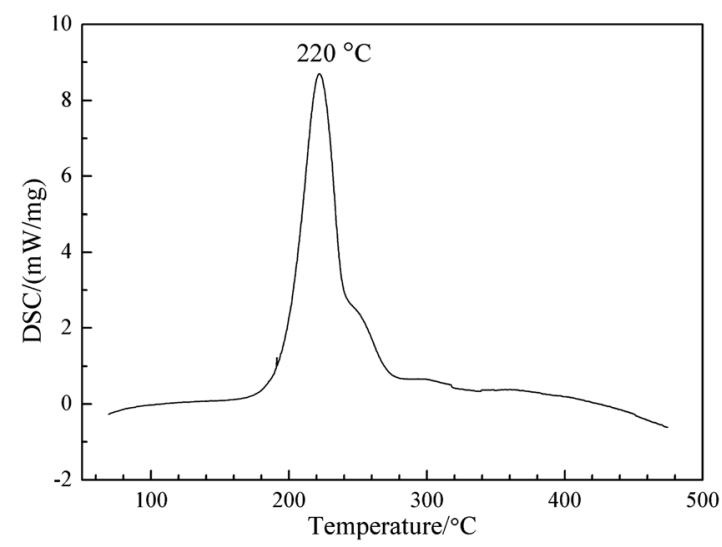

Fig. 8 DSC curve of PBFMO- $b$-PNMMO.

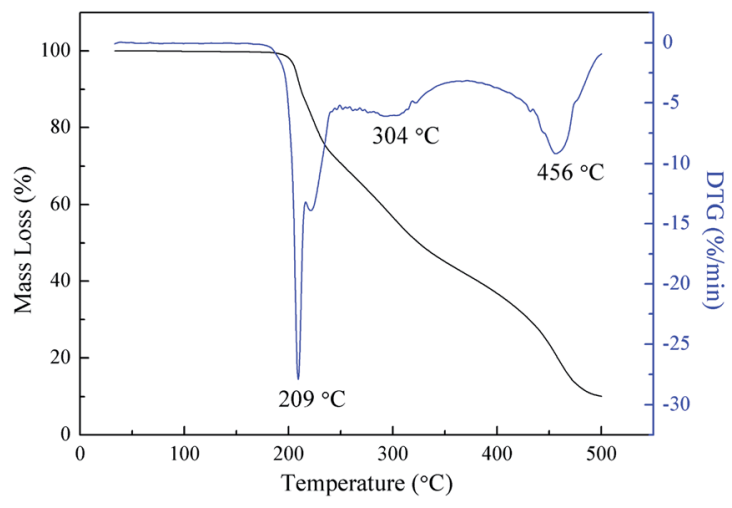

Fig. 9 TGA curves of PBFMO- $b$-PNMMO.

heat release due to the reaction between elastomers and $\mathrm{Al}$. In this work, the integral of the PBFMO- $b$-PNMMO/Al group showed a remarkable increase than that of the control group. This implies that PBFMO- $b$-PNMMO can efficiently react with $\mathrm{Al}$ and release significantly more heat. ${ }^{36}$

\subsection{Compatibility testing}

The compatibility of binders and explosives plays a predominant role in the thermal stability of solid propellant formulations. ${ }^{37,38}$ In this study, the compatibility of PBFMO- $b$-PNMMO
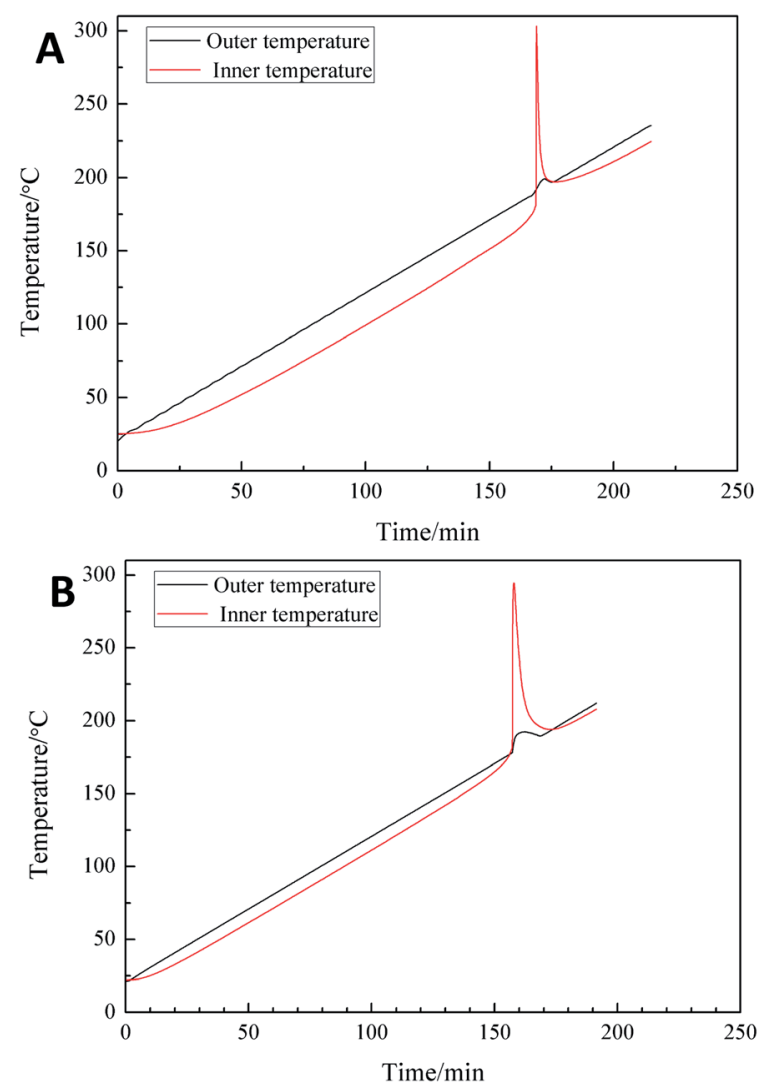

Fig. 10 Cook-off curves of PNMMO-ETPE/Al compositions (A) and PBFMO- $b$-PNMMO/Al compositions (B). 


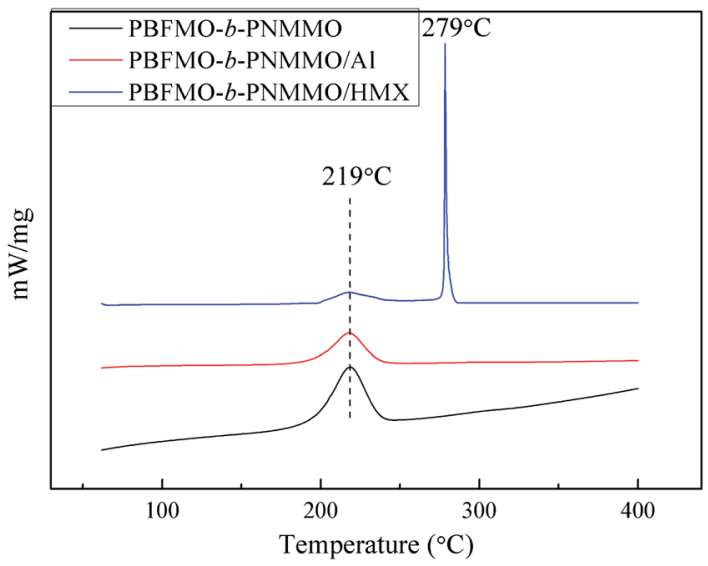

Fig. 11 DSC curves of PBFMO- $b$-PNMMO, PBFMO- $b$-PNMMO/HMX compositions and PBFMO- $b$-PNMMO/Al compositions.

with the main energetic components (viz., HMX and Al) was tested by studying the effect of the contact materials on the exothermic decomposition temperature of the explosives from the DSC curves. As shown in Fig. 11, the $\Delta T_{\mathrm{p}}$ values of the binary systems, i.e., PBFMO- $b$-PNMMO/HMX and PBFMO- $b$-PNMMO/ $\mathrm{Al}$ are all less than $2{ }^{\circ} \mathrm{C}$. The results indicate that PBFMO- $b$ PNMMO has good compatibilities with HMX and $\mathrm{Al}$ and can be safely used in HMX-based Al-rich propellants.

\section{Conclusions}

In this study, a novel energetic polymer, PBFMO- $b$-PNMMO, was synthesized using PNMMO and PBFMO as the raw materials and TDI as the coupling agent via a prepolymer process. The structure of PBFMO- $b$-PNMMO was confirmed by FTIR, ${ }^{1} \mathrm{H}$ NMR and ${ }^{13} \mathrm{C}$ NMR. The PBFMO- $b$-PNMMO films exhibited a superior mechanical performance with a tensile strength of $10.54 \mathrm{MPa}$ and the corresponding breaking elongation of $723 \%$. The DSC and TGA results indicated that PBFMO- $b$-PNMMO exhibited $T_{\mathrm{g}}$ of $-20.4{ }^{\circ} \mathrm{C}$ and a good resistance to thermal decomposition up to $200{ }^{\circ} \mathrm{C}$. The cook-off results indicated that PBFMO- $b$-PNMMO can effectively react with $\mathrm{Al}$ and release significantly more heat. Moreover, PBFMO- $b$-PNMMO has good compatibilities with the main components in propellants. All these results confirm that PBFMO- $b$-PNMMO might serve as a new energetic binder in propellant formulations.

\section{Conflicts of interest}

There are no conflicts to declare.

\section{Acknowledgements}

The authors gratefully acknowledge the financial support from the China Postdoctoral Science Foundation (2016M592851).

\section{Notes and references}

1 T. Cheng, Des. Monomers Polym., 2019, 22, 54-65.
2 A. K. Sikder and S. Reddy, Propellants, Explos., Pyrotech., 2013, 38, 14-28.

3 A. Bodaghi and M. Shahidzadeh, Propellants, Explos., Pyrotech., 2018, 43, 364-370.

4 Y. Hu, X. Jian, L. Xiao and W. Zhou, Polym. Eng. Sci., 2018, 58, 167-173.

5 S. Hafner, T. Keicher and T. M. Klapoetke, Propellants, Explos., Pyrotech., 2018, 43, 126-135.

6 F. Abrishami, N. Zohari and V. Zeynali, Polym. Adv. Technol., 2019, 30, 640-647.

7 X. Wang, X. Lu, Y. Shu, H. Mo, M. Xu and N. Liu, Fine Chem., 2019, 36, 348-353.

8 C. Mura, S. Fruci, P. Lamia, M. Cappello, S. Filippi and G. Polacco, J. Energ. Mater., 2016, 34, 216-233.

9 S. K. Valluri, M. Schoenitz and E. L. Dreizin, J. Mater. Sci., 2017, 52, 7452-7465.

10 O. Eisenstein, J. Milani and R. N. Perutz, Chem. Rev., 2017, 117, 8710-8753.

11 J. H. Lee, S. J. Kim, J. S. Park and J. H. Kim, Macromol. Res., 2016, 24, 909-914.

12 F. Gong, H. Guo, J. Zhang, C. Shen, C. Lin, C. Zeng and S. Liu, Propellants, Explos., Pyrotech., 2017, 42, 1424-1430.

13 H. Yang, C. Huang and H. Chen, J. Therm. Anal. Calorim., 2017, 127, 2293-2299.

14 D. M. Dattelbaum, S. A. Sheffield, D. Stahl, M. Weinberg, C. Neel and N. Thadhani, J. Appl. Phys., 2008, 104, 113525113535.

15 K. B. Rider, B. K. Little, S. B. Emery and C. M. Lindsay, Propellants, Explos., Pyrotech., 2013, 38, 433-440.

16 W. C. Jiang, Y. G. Huang, G. T. Gu, W. D. Meng and F. L. Qing, Appl. Surf. Sci., 2006, 253, 2304-2309.

17 H. Mo, X. Lu, L. Li, M. Chen, Y. Ji and W. Wang, Chin. J. Energetic Mater., 2015, 23, 629-632.

18 M. O. Hongchang, G. A. N. Xiaoxian, X. Ying and L. I. Na, Chin. J. Explos. Propellants, 2008, 31, 24-27.

19 Q. Dong, H. Li, X. Liu and C. Huang, Propellants, Explos., Pyrotech., 2018, 43, 294-299.

20 X. Liu, H. Gao, X. Chen, Y. Hu, S. Pei, H. Li and Y. Zhang, J. Membr. Sci., 2016, 515, 268-276.

21 X. Wang, J. Hu, Y. Li, J. Zhang and Y. Ding, J. Fluorine Chem., 2015, 176, 14-19.

22 M. H. Xu, Z. X. Ge, X. M. Lu, H. C. Mo, Y. P. Ji and H. M. Hu, Polym. Int., 2017, 66, 1318-1323.

23 M. Ma and Y. Kwon, Polym. Chem., 2018, 9, 5452-5461.

24 A. Tanver, F. Rehman, A. Wazir, S. Khalid, S. Ma, X. Li, Y. Luo and M. H. Huang, RSC Adv., 2016, 6, 11032-11039.

25 I. K. Boshra, A. Elbeih and H. E. Mostafa, Z. Anorg. Allg. Chem., 2019, 645, 551-557.

26 Y. Yanagisawa, Y. Nan, K. Okuro and T. Aida, Science, 2018, 359, 72-79.

27 K. Malkappa and T. Jana, Ind. Eng. Chem. Res., 2013, 52, 12887-12896.

28 M. Chizari and Y. Bayat, Cent. Eur. J. Energ. Mater., 2018, 15, 243-257.

29 Y. Wu, Z. Yi, Y. Luo, Z. Ge, F. Du, S. Chen and J. Sun, J. Therm. Anal. Calorim., 2017, 129, 1555-1562. 
30 M. Byoung Sun and K. Seung Won, Macromol. Res., 2007, 15, 225-233.

31 E. Landsem, T. L. Jensen, T. E. Kristensen, F. K. Hansen, T. Benneche and E. Unneberg, Propellants, Explos., Pyrotech., 2013, 38, 75-86.

32 J. S. You, J. O. Kweon, S. C. Kang and S. T. Noh, Macromol. Res., 2010, 18, 1226-1232.

33 X. Y. Ding, Y. J. Shu, H. T. Xu and Z. Q. Chen, Propellants, Explos., Pyrotech., 2018, 43, 267-273.

34 W. F. Li, Y. G. Yu, R. Ye and H. W. Yang, J. Energ. Mater., 2017, 35, 265-275.
35 L. Chen, X. Ma, F. Lu and J. Y. Wu, Cent. Eur. J. Energ. Mater., 2014, 11, 199-218.

36 M. H. Xu, Z. X. Ge, X. M. Lu, H. C. Mo, Y. P. Ji and H. M. Hu, RSC Adv., 2017, 7, 47271-47278.

37 Y. Li, J. Li, S. Ma and Y. Luo, Polym. Bull., 2017, 74, 46074618.

38 J. F. Pei, F. Q. Zhao, H. L. Lu, X. D. Song, R. Zhou, Z.-F. Yuan, J. Zhang and J. B. Chen, J. Therm. Anal. Calorim., 2016, 124, 1301-1307. 